Journal of Mathematics and Statistics 7 (3): 230-238, 2011

ISSN 1549-3644

(C) 2011 Science Publications

\title{
An Approximate Formula of European Option for Fractional Stochastic Volatility Jump-Diffusion Model
}

\author{
${ }^{1}$ P. Sattayatham and ${ }^{2}$ A. Intarasit \\ ${ }^{1}$ Institute of Science, School of Mathematics, \\ Suranaree University of Technology, Thailand \\ ${ }^{2}$ Department of Mathematics and Computer Science, \\ Faculty of Science and Technology, Prince of Songkla University, \\ Pattani Campus, Thailand
}

\begin{abstract}
Problem statement: We presented option pricing when the stock prices follows a jumpdiffusion model and their stochastic volatility follows a fractional stochastic volatility model. This proposed model exhibits the a memory of a stochastic volatility model that is not expressed in the classical stochastic volatility model. Approach: We introduce an approximated method to fractional stochastic volatility model perturbed by the fractional Brownian motion. A relationship between stochastic differential equations and partial differential equations for a bivariate model is presented. Results: By using an approximate method, we provide the approximate solution of the fractional stochastic volatility model. And European options are priced by using the risk-neutral valuation. Conclusion/Recommendations: The formula of European option is calculated by using the technique base on the characteristic function of an underlying asset which can be expressed in an explicit formula. A numerical integration technique to simulation fractional stochastic volatility are presented in this study.
\end{abstract}

Key words: Fractional Brownian motion, approximate method, fractional stochastic volatility, jump diffusion model, option pricing model

\section{INTRODUCTION}

Let $(\Omega, F, P)$ be a probability space with filtration $\mathbb{F}=\left(F_{t}\right)_{0 \leq t \leq T}$. All processes that we shall consider in this section will be defined in this space. For $t \in[0, \mathrm{~T}]$ and $\mathrm{T}<\infty$ a geometric Brownian motion $(\mathrm{gBm})$ model with jumps and with fractional stochastic volatility is a model of the form:

$\mathrm{dS}_{\mathrm{t}}=\mathrm{S}_{\mathrm{t}}\left(\mu \mathrm{dt}+\sqrt{\mathrm{v}_{\mathrm{t}}} \mathrm{dW}_{\mathrm{t}}\right)+\mathrm{S}_{\mathrm{t}-} \mathrm{YdN}_{\mathrm{t}}$

where $\mu \in \Re, S=\left(S_{t}\right)_{t \in[0, T]}$ is a process representing a price of the underlying risky assets, $\mathrm{W}=\left(\mathrm{W}_{\mathrm{t}}\right)_{\mathrm{t} \in[0, \mathrm{~T}]}$ is the standard Brownian motion, $\mathrm{N}=\left(\mathrm{N}_{\mathrm{t}}\right)_{\mathrm{t} \in[0, \mathrm{~T}]}$ is a Poisson process with intensity $\lambda$ and $S_{t} Y_{t}$ represents the amplitude of the jump which occurs at time t. We assume that the processes $\mathrm{W}$ and $\mathrm{N}$ are independent. The volatility process $\mathrm{v}_{\mathrm{t}}:=\sigma_{\mathrm{t}}^{2}$ in (1) is modeled by:

$d v_{t}=\left(\omega-\theta v_{t}\right) d t+\xi v_{t} d B_{t}$ where, $\omega>0$ is the mean long-term volatility, $\theta \in \Re$ is the rate at which the volatility reverts toward its longterm mean, $\xi>0$ is the volatility of the volatility process and $\left(\mathrm{B}_{\mathrm{t}}\right)_{\mathrm{t} \in[0, \mathrm{~T}]}$ is a fractional Brownian motion.

Assume that the processes $\left(\mathrm{S}_{\mathrm{t}}\right)$ and $\left(\mathrm{v}_{\mathrm{t}}\right)$ are $\mathrm{F}_{\mathrm{t}^{-}}$ measurable.

The notation $\mathrm{S}_{\mathrm{t}}$ means that whenever there is a jump, the value of the process before the jump is used on the left-hand side of the formula.

The fractional version of Eq. 1 is given by:

$\mathrm{dS}_{\mathrm{t}}=\mathrm{S}_{\mathrm{t}}\left(\mu \mathrm{dt}+\sqrt{\mathrm{v}_{\mathrm{t}}} \mathrm{dB}_{\mathrm{t}}\right)+\mathrm{S}_{\mathrm{t}-} \mathrm{Y}_{\mathrm{t}} \mathrm{dN}_{\mathrm{t}}$

The process $S_{t}$ in (3) can be approximated in $\mathrm{L}_{2}(\Omega)$ by a semimartingale $\mathrm{S}_{\mathrm{t}}^{\varepsilon}$ in the sense that $\left\|\mathrm{S}_{\mathrm{t}}^{\varepsilon}-\mathrm{S}_{\mathrm{t}}\right\|_{\mathrm{L}_{2}(\Omega)} \rightarrow 0$ as $\varepsilon \rightarrow 0$, where $\mathrm{S}_{\mathrm{t}}^{\varepsilon}$ satisfies the following equation (Intarasit and Sattayatham, 2010 for more details):

$$
\mathrm{dS}_{\mathrm{t}}^{\varepsilon}=\mathrm{S}_{\mathrm{t}}^{\varepsilon}\left(\mu \mathrm{dt}+\sqrt{\mathrm{v}_{\mathrm{t}}^{\varepsilon}} \mathrm{dB}_{\mathrm{t}}^{\varepsilon}\right)+\mathrm{S}_{\mathrm{t}-}^{\varepsilon} \mathrm{Y}_{\mathrm{t}} \mathrm{dN}_{\mathrm{t}}
$$


The purpose of this study is to consider the problem of option pricing for the $\mathrm{gBm}$ model with jumps (1) and with fractional stochastic volatility (2). Since driving process $B_{t}$ of $v_{t}$ in Eq. 2 is not a semimartingale, thus we cannot apply Itô calculus directly. We shall thus work in another direction by introducing an approximate model of SDE (1) and (2) then using it to price European call option. The advantage of these approximate model is there no more arbitrage. In order to find such a formula, we shall work in the space of a risk-neutral probability measure. Indeed, there are some authors who have investigated this problem before but not in the fractional case, for example (Heston, 1993). In fact, there are many author studies a volatility and fractional volatility process. For example (Magnus and Fosu, 2006) use GARCH to model and forecast volatility returns on the Ghana stock exchange and (Shamiri and Isa, 2009) study modeling and forecasting of volatility of the Malaysian stock markets. An empirical study of fractional volatility are presented in (Cheong, 2008) for example.

Recall that the fractional Brownian motion with Hurst coefficient is a Gaussian process $B^{\mathrm{H}}=\left(\mathrm{B}_{t}^{\mathrm{H}}\right)_{t \geq 0}$ with zero mean and the covariance function is given by:

$\mathrm{R}(\mathrm{t}, \mathrm{s})=\mathrm{E}\left[\mathrm{B}_{\mathrm{t}}^{\mathrm{H}} \mathrm{B}_{\mathrm{s}}^{\mathrm{H}}\right]=\frac{1}{2}\left(\mathrm{~s}^{2 \mathrm{H}}+\mathrm{t}^{2 \mathrm{H}}-|\mathrm{t}-\mathrm{s}|^{2 \mathrm{H}}\right)$

If $H=1 / 2$, then $R(t, s)=\min (t, s)$ and $B_{t}^{H}$ is the usual standard Brownian motion. In the case $1 / 2<\mathrm{H}<$ 1 the fractional Brownian motion exhibits statistical long-range dependency in the sense that $\rho_{\mathrm{n}}:=\mathrm{E}\left[\mathrm{B}_{1}^{\mathrm{H}}\left(\mathrm{B}_{\mathrm{n}+1}^{\mathrm{H}}-\mathrm{B}_{\mathrm{n}}^{\mathrm{H}}\right)\right]>0$ for all $\mathrm{n}=1,2,3, \ldots$ and $\sum_{n=1}^{\infty} \rho_{n}=\infty$. Hence, in financial modeling, one usually assumes that $H \in(1 / 2,1)$. Put $\alpha=1 / 2-H$. It is known that a fractional Brownian motion $\mathrm{B}_{\mathrm{t}}^{\mathrm{H}}$ can be decomposed as follows:

$\mathrm{B}_{\mathrm{t}}^{\mathrm{H}}=\frac{1}{\Gamma(1+\alpha)}\left\{\mathrm{Z}_{\mathrm{t}}+\int_{0}^{\mathrm{t}}(\mathrm{t}-\mathrm{s})^{-\alpha} \mathrm{dW}_{\mathrm{s}}\right\}$

where, $\Gamma$ is the gamma function:

$Z_{t}=\int_{-\infty}^{0}\left[(t-s)^{-\alpha}-(s)^{-\alpha}\right] d W_{s}$

We suppose from now on that $0<\alpha<1 / 2$. The process $Z_{t}$ has absolutely continuous trajectories, so it suffices to consider only the term:
$\mathrm{B}_{\mathrm{t}}=\int_{0}^{\mathrm{t}}(\mathrm{t}-\mathrm{s})^{-\alpha} \mathrm{dW}_{\mathrm{s}}$

that has a long-range dependence.

Note that $\mathrm{B}_{\mathrm{t}}$ can be approximated by:

$\mathrm{B}_{\mathrm{t}}^{\varepsilon}=\int_{0}^{\mathrm{t}}(\mathrm{t}-\mathrm{s}+\varepsilon)^{-\alpha} \mathrm{dw} \mathrm{s}_{\mathrm{s}}$

in the sense that $\mathrm{B}_{t}^{\varepsilon}$ converges to $\mathrm{B}_{\mathrm{t}}$ in $\mathrm{L}_{2}(\Omega)$ as $\varepsilon \rightarrow 0$, uniform with respect to $t \in[0, T]$ (Thao, 2006).

Since $\left(B_{t}^{\varepsilon}\right)_{t \in[0, T]}$ is a continuous semimartingale then Itô calculus can be applied to the following Stochastic Differential Equation (SDE):

$$
\mathrm{dS}_{\mathrm{t}}^{\varepsilon}=\mathrm{S}_{\mathrm{t}}^{\varepsilon}\left(\mu \mathrm{dt}+\sigma \mathrm{dB}_{\mathrm{t}}^{\varepsilon}\right), 0 \leq \mathrm{t} \leq \mathrm{T}
$$

Let $S_{t}^{\varepsilon}$ be the solution of the above equation. Because of the convergence of $B_{t}^{\varepsilon}$ to $B_{t}$ in $L_{2}(\Omega)$ when $\varepsilon \rightarrow 0$, we shall define the solution of a fractional stochastic differential equation of the form:

$\mathrm{dS}_{\mathrm{t}}=\mathrm{S}_{\mathrm{t}}\left(\mu \mathrm{dt}+\sigma \mathrm{dB}_{\mathrm{t}}\right), 0 \leq \mathrm{t} \leq \mathrm{T}$

to be a process $S_{t}^{*}$ defined on the probability space $(\Omega$, $\mathrm{F}, \mathrm{P})$ such that the process $\mathrm{S}_{\mathrm{t}}^{\varepsilon}$ converges to $\mathrm{S}_{\mathrm{t}}^{*}$ in $\mathrm{L}_{2}(\Omega)$ as $\varepsilon \rightarrow 0$ and the convergence is uniform with respect to $t \in[0, T]$. This definition will be applied to the other similar fractional stochastic differential equations which will appear later.

A risk-neutral model for a $\mathrm{gBm}$ model combining jumps with stochastic volatility is introduced next. Its solution will also be discussed. Firstly, let us rewrite the model (1) into an integral form as follows:

$$
\mathrm{S}_{\mathrm{t}}=\mathrm{S}_{0}+\int_{0}^{\mathrm{t}} \mu \mathrm{S}_{\mathrm{s}} \mathrm{ds}+\int_{0}^{\mathrm{t}} \sqrt{\mathrm{v}_{\mathrm{s}}} \mathrm{S}_{\mathrm{s}} \mathrm{dW} \mathrm{W}_{\mathrm{s}}+\int_{0}^{\mathrm{t}} \mathrm{S}_{\mathrm{s}-} \mathrm{Y}_{\mathrm{s}} \mathrm{dN} \mathrm{N}_{\mathrm{s}}
$$

Note that the last term on the right hand side of Eq. 6 is defined by:

$\int_{0}^{\mathrm{t}} \mathrm{S}_{\mathrm{s}-} \mathrm{Y}_{\mathrm{s}} \mathrm{dN} \mathrm{N}_{\mathrm{s}}:=\sum_{\mathrm{n}=1}^{\mathrm{N}_{\mathrm{t}}} \Delta \mathrm{S}_{\mathrm{n}}$

Where:

$$
\Delta \mathrm{S}_{\mathrm{n}}:=\mathrm{ST}_{\mathrm{n}}-\mathrm{ST}_{\mathrm{n}-}=\mathrm{S}_{\mathrm{n}-} \mathrm{Y}_{\mathrm{n}}
$$


The assumption $Y_{n}>0$ always leads to positive values of the stock prices. The process $\left(Y_{n}\right)_{n \in N}$ is assumed to be independently identically distributed (i.i.d.) with density $\phi_{Y}(y)$ and $\left(T_{n}\right)_{n \in N}$ is a sequence of jump time.

In order to solve Eq. 6 with an initial condition $\mathrm{S}_{\mathrm{t}(\mathrm{t}=0)}=\mathrm{S}_{0}$ we assume that $\mathrm{E}\left[\int_{0}^{\mathrm{T}} \mathrm{v}_{\mathrm{s}} \mathrm{S}_{\mathrm{s}}^{2} \mathrm{ds}\right]<\infty$. Then, by an application of Itô's formula for the jump process (Cont and Tankov, 2009, Theorem 8.14) on Eq. 6 with $\mathrm{f}\left(\mathrm{S}_{\mathrm{t}}, \mathrm{t}\right)=\log \left(\mathrm{S}_{\mathrm{t}}\right)$ we get:

$\mathrm{S}_{\mathrm{t}}=\mathrm{S}_{0} \exp \left(\mu \mathrm{t}-\frac{1}{2} \int_{0}^{\mathrm{t}} \mathrm{v}_{\mathrm{s}} \mathrm{ds}+\int_{0}^{\mathrm{t}} \sqrt{\mathrm{v}_{\mathrm{s}}} \mathrm{dW} \mathrm{W}_{\mathrm{s}}+\int_{0}^{\mathrm{t}} \log \left(1+\mathrm{Y}_{\mathrm{s}}\right) \mathrm{dN}_{\mathrm{s}}\right)$

It is assumed that a risk-neutral probability measure $M$ exists; the asset price $S_{t}$, under this riskneutral measure, follows a jump-diffusion process, with zero-mean, risk-free rate $\mathrm{r}$ :

$\left.d S_{t}=S_{t}\left(r-\lambda E_{M}\left[Y_{t}\right]\right) d t+\sqrt{v_{t}} d W_{t}\right)+S_{t-} Y_{t} d N_{t}$

and the stochastic variance $\mathrm{v}_{\mathrm{t}}$ satisfies the following fractional SDE:

$d v_{t}=\left(\omega-\theta v_{t}\right) d t+\xi v_{t} d B_{t}$

with an initial condition $\mathrm{v}_{\mathrm{t}(\mathrm{t}=0)}=\mathrm{v}_{0} \in \mathrm{L}_{2}(\Omega)$.

It is only necessary to know that the risk-neutral measure exists (Cont and Tankov, 2009). Hence, all processes to be discussed after this will be the processes under the risk-neutral probability measure $\mathrm{M}$.

Using an initial condition $\mathrm{S}_{\mathrm{t}(\mathrm{t}=0)}=\mathrm{S}_{0} \in \mathrm{L}_{2}(\Omega)$, the solution of Eq. 7 is given by:

$\mathrm{S}_{\mathrm{t}}=\mathrm{S}_{0} \exp \left(\begin{array}{l}\int_{0}^{\mathrm{t}}\left(\mathrm{r}-\lambda \mathrm{E}_{\mathrm{M}}\left[\mathrm{Y}_{\mathrm{s}}\right]\right) \mathrm{ds}-\frac{1}{2} \int_{0}^{\mathrm{t}} \mathrm{v}_{\mathrm{s}} \mathrm{ds}+\int_{0}^{\mathrm{t}} \sqrt{\mathrm{V}_{\mathrm{s}}} \mathrm{dW} \\ +\int_{0}^{\mathrm{t}} \log \left(1+\mathrm{Y}_{\mathrm{s}}\right) \mathrm{dN}\end{array}\right)$.

Under approximate method, for each $\varepsilon>0$, consider an approximate model of Eq. 7 and 8 respectively:

$$
\begin{aligned}
& d S_{t}^{\varepsilon}=S_{t}^{\varepsilon}\left(\left(r-\lambda E_{M}\left[Y_{t}\right]\right) d t+\sqrt{v_{t}^{\varepsilon}} W_{t}\right)+S_{t-}^{\varepsilon} Y_{t} d N_{t} \\
& d v_{t}^{\varepsilon}=\left(\omega-\theta v_{t}^{\varepsilon}\right) d t+\xi v_{t}^{\varepsilon} d B_{t}^{\varepsilon}
\end{aligned}
$$

By using the same initial condition as in Eq. 10, we have:

$$
S_{t}^{\varepsilon}=S_{0} \exp \left(\begin{array}{l}
\int_{0}^{t}\left(r-\lambda E_{M}\left[Y_{s}\right]\right) d s-\frac{1}{2} \int_{0}^{t} v_{s}^{\varepsilon} d s+ \\
\int_{0}^{t} \sqrt{v_{s}^{\varepsilon}} d W_{s}+\int_{0}^{t} \log \left(1+Y_{s} d N_{s}\right)
\end{array}\right)
$$

and one can prove that $S_{t}^{\varepsilon}$ converges to $S_{t}$ of Eq. 9 in $\mathrm{L}_{2}(\Omega)$ as $\varepsilon \rightarrow 0$ and uniformly on $\mathrm{t} \in[0, \mathrm{~T}]$. Moreover, one can show that the solution $v_{t}^{\varepsilon}$ of Eq. 11 converges in $\mathrm{L}_{2}(\Omega)$ to the process:

$$
\mathrm{v}_{\mathrm{t}}=\left(\mathrm{v}_{0}+\omega \int_{0}^{\mathrm{t}} \exp \left(\gamma \mathrm{s}-\xi \mathrm{B}_{\mathrm{s}}\right) \mathrm{ds}\right) \exp \left(\xi \mathrm{B}_{\mathrm{t}}-\gamma \mathrm{t}\right)
$$

for some real constant $\gamma$. Hence, by definition, $\mathrm{v}_{\mathrm{t}}$ is the solution of Eq. 8 (Intarasit and Sattayatham, 2010, Lemma 2).

\section{MATERIALS AND METHODS}

The relationship between the stochastic deferential equation and the partial differential equation for bivarate model is presented.

Consider the process $\vec{X}_{t}=\left(X_{t}^{1}, X_{t}^{2}\right)$ where $X_{t}^{1}$ and $\mathrm{X}_{\mathrm{t}}^{2}$ are processes in $\mathfrak{R}$ and satisfy the following equations:

$$
\begin{aligned}
& d X_{t}^{1}=f_{1}(t) d t+g_{1}(t) d W_{t}+X_{t-}^{1} Y_{t} d N_{t} \\
& d X_{t}^{2}=f_{2}(t) d t+g_{2}(t) d \bar{W}_{t}
\end{aligned}
$$

where, $f_{1}, g_{1}, f_{2}$ and $g_{2}$ are all continuous functions from $[0, \mathrm{~T}]$ into $\mathfrak{R}$.

Since every compound Poisson process can be represented as an integral form of Poisson random measure (Cont and Tankov, 2009) then the last term on the right hand side of Eq. 13 can be written as follows:

$$
\begin{aligned}
& \int_{0}^{t} X_{s-}^{1} Y_{s} d N_{s}=\sum_{n=1}^{N_{t}} X_{n-}^{1} Y_{n}=\sum_{n=1}^{N_{t}}\left[X_{T_{n}}^{1}-X_{T_{n}-}^{1}\right] \\
& =\int_{0}^{t} \int_{\Re} X_{s-}^{1} Z J_{z}(d s d z)
\end{aligned}
$$

where, $Y_{n}$ are i.i.d. random variables with density $\phi_{Y}$ (y) and $J_{Z}$ is a Poisson random measure of the process $Z_{t}=\sum_{n=1}^{N_{t}} Y_{n}$ with intensity measure $\lambda \phi_{Y}\left(d_{z}\right) d t$. 
Let $U(\vec{x})$ be a bounded real function on $\Re^{2}$ and twice continuously differentiable in $\overrightarrow{\mathrm{x}}=\left(\mathrm{x}_{1}, \mathrm{x}_{2}\right) \in \mathfrak{R}^{2}$ and:

$$
\mathrm{u}(\overrightarrow{\mathrm{x}}, \mathrm{t})=\mathrm{E}\left[\mathrm{u}\left(\overrightarrow{\mathrm{X}}_{\mathrm{T}}\right) \overrightarrow{\mathrm{X}}_{\mathrm{t}}=\overrightarrow{\mathrm{x}}\right]
$$

By the two dimensional Dynkin's formula (Hanson, 2007, Theorem 7.7), $\mathrm{u}$ is a solution of the Partial Integro-Differential Equation (PIDE):

$0=\frac{\partial v(\vec{x}, t)}{\partial t}+\operatorname{Av}(\vec{x}, t)+\lambda \int_{\Re}[v(\vec{x}+\vec{y}, t)-v(\vec{x}, t)] \phi_{Y}(y) d y$

subject to the final condition $\mathrm{u}(\overrightarrow{\mathrm{x}}, \mathrm{T})=\mathrm{U}(\overrightarrow{\mathrm{x}})$ and $\vec{y}=(y, 0)$. The notation A is defined by:

$$
\begin{aligned}
& \operatorname{Au}(\vec{x}, t)=f_{1}(t) \frac{\partial u(\vec{x}, t)}{\partial x_{1}}+f_{2}(t) \frac{\partial u(\vec{x}, t)}{\partial x_{2}}+\frac{1}{2} g_{1}^{2}(t) \frac{\partial^{2} u(\vec{x}, t)}{\partial x_{1}^{2}} \\
& +\rho g_{1}(t) g_{2}(t) \frac{\partial^{2} u(\vec{x}, t)}{\partial x_{1} \partial x_{2}}+\frac{1}{2} g_{2}^{2}(t) \frac{\partial^{2} u(\vec{x}, t)}{\partial x_{2}^{2}}
\end{aligned}
$$

and the correlation $\rho$ defined by $\rho=\operatorname{Corr}\left[\mathrm{dW}_{t}, \mathrm{dW}_{t}\right]$.

Next, we present the classical method to pricing of European call option. The European call option formula in terms of characteristic function is given in the next section.

Let $\mathrm{C}$ denote the price at time $\mathrm{t}$ of a European style call option on the current price of the underlying asset $\mathrm{S}_{\mathrm{t}}$ with strike price $\mathrm{K}$ and expiration time $\mathrm{T}$. The terminal payoff of a European call option on the underling stock $S_{t}$ with strike price $K$ is $\max \left(S_{T^{-}} K ; 0\right)$. This means that the holder will exercise his right only if $\mathrm{S}_{\mathrm{T}}>\mathrm{K}$ and then his gain is $\mathrm{S}_{\mathrm{T}}-\mathrm{K}$. Otherwise, if $\mathrm{S}_{\mathrm{T}}>\mathrm{K}$, then the holder will buy the underlying asset from the market and the value of the option is zero. Assuming the risk-free interest rate $r$ is constant over the lifetime of the option, the price of the European call at time $t$ is equal to the discounted conditional expected payoff:

$$
\begin{aligned}
& \mathrm{C}\left(\mathrm{S}_{\mathrm{t}}, \mathrm{v}_{\mathrm{t}}, \mathrm{t} ; \mathrm{K}, \mathrm{T}\right)=\mathrm{e}^{-\mathrm{r}(\mathrm{T}-\mathrm{t})} \mathrm{E}_{\mathrm{M}}\left[\max \left(\mathrm{S}_{\mathrm{T}}-\mathrm{K}, 0\right) \mid \mathrm{S}_{\mathrm{t}}, \mathrm{v}_{\mathrm{t}}\right] \\
& =\mathrm{e}^{-\mathrm{r}(\mathrm{T}-\mathrm{t})}\left(\int_{\mathrm{K}}^{\infty}\left(\mathrm{S}_{\mathrm{T}}-\mathrm{K}\right) \mathrm{P}_{\mathrm{M}}\left(\mathrm{S}_{\mathrm{T}} \mid \mathrm{S}_{\mathrm{t}}, \mathrm{v}_{\mathrm{t}}\right) \mathrm{d} \mathrm{S}_{\mathrm{T}}\right) \\
& =S_{t}\left(\frac{1}{E_{M}\left[S_{T} \mid S_{t}, t\right]} \int_{K}^{\infty} S_{T} P_{M}\left(S_{T} \mid S_{t}, v_{t}\right) d S_{T}\right) \\
& -\mathrm{Ke}^{-\mathrm{r}(\mathrm{T}-1)} \int_{\mathrm{K}}^{\infty} \mathrm{P}_{\mathrm{M}}\left(\mathrm{S}_{\mathrm{T}} \mid \mathrm{S}_{\mathrm{t}}, \mathrm{v}_{\mathrm{t}}\right) \mathrm{d} \mathrm{S}_{\mathrm{T}} \\
& =S_{t} P_{1}\left(S_{t}, v_{t}, t ; K<T\right)-K e^{-r(T-t)} P_{2}\left(S_{t}, v_{t}, t ; K<T\right)
\end{aligned}
$$

where, $E_{M}$ is the expectation with respect to the riskneutral probability measure, $\mathrm{P}_{\mathrm{M}}\left(\mathrm{S}_{\mathrm{T}} \mid \mathrm{S}_{\mathrm{t}}, \mathrm{v}_{\mathrm{t}}\right)$ is the corresponding conditional density given $\left(\mathrm{S}_{\mathrm{t}}, \mathrm{v}_{\mathrm{t}}\right)$ and:

$$
\mathrm{P}_{1}\left(\mathrm{~S}_{\mathrm{t}}, \mathrm{v}_{\mathrm{t}}, \mathrm{t} ; \mathrm{K}, \mathrm{T}\right)=\left(\int_{\mathrm{K}}^{\infty} \mathrm{S}_{\mathrm{T}} \mathrm{P}_{\mathrm{M}}\left(\mathrm{S}_{\mathrm{T}} \mid \mathrm{S}_{\mathrm{t}}, \mathrm{v}_{\mathrm{t}}\right) \mathrm{d} \mathrm{S}_{\mathrm{T}}\right) / \mathrm{E}_{\mathrm{M}}\left[\mathrm{S}_{\mathrm{T}} \mid \mathrm{S}_{\mathrm{t}}, \mathrm{v}_{\mathrm{t}}\right]
$$

Note that $P_{1}$ is the risk-neutral probability that $S_{T}>$ $\mathrm{K}$ (since the integrand is nonnegative and the integral over $[0, \infty)$ is one) and finally, that:

$$
\mathrm{P}_{2}\left(\mathrm{~S}_{\mathrm{t}}, \mathrm{v}_{\mathrm{t}}, \mathrm{t} ; \mathrm{K}, \mathrm{T}\right)=\int_{\mathrm{K}}^{\infty} \mathrm{P}_{\mathrm{M}}\left(\mathrm{S}_{\mathrm{t}}, \mathrm{v}_{\mathrm{t}}\right) \mathrm{dS} \mathrm{S}_{\mathrm{T}}=\operatorname{Prob}\left(\mathrm{S}_{\mathrm{T}}>\mathrm{K} \mid \mathrm{S}_{\mathrm{t}}, \mathrm{v}_{\mathrm{t}}\right)
$$

is the risk-neutral in-the-money probability. Moreover, $E_{M}\left[S_{T} \mid S_{t}, v_{t}\right]=e^{r(T-t)} S_{t}$ for $t \geq 0$.

Note that we do not have a formulation for these probabilities thus we will calculate some approximations of $\mathrm{P}_{1}$ and $\mathrm{P}_{2}$. Indeed, these probabilities are related to characteristic functions which have formulation as will be seen in Lemma 2 .

\section{RESULTS}

In order to calculate the price of a European call option with strike price $\mathrm{K}$ and maturity $\mathrm{T}$ of the model (7) for which its fractional stochastic volatility satisfies Eq. 8, we consider the approximate model (10) and (11). Firstly, we consider logarithm of $\mathrm{S}_{\mathrm{t}}^{\varepsilon}$ namely $\mathrm{L}_{\mathrm{t}}^{\varepsilon}$, i.e. $\mathrm{L}_{\mathrm{t}}^{\varepsilon}=\log \left(\mathrm{S}_{\mathrm{t}}^{\varepsilon}\right)$ where $\mathrm{S}_{\mathrm{t}}^{\varepsilon}$ satisfies Eq. 12 (the solution of Eq. 10) and its inverse $S_{t}^{\varepsilon}=\exp \left(L_{t}^{\varepsilon}\right)$. Denote $\mathrm{k}=\log (\mathrm{K})$ the logarithm of the strike price. Secondly, we now refer to $\operatorname{SDE}(11)$, since this approximate model is driven by a semimartingale $B_{t}^{\varepsilon}$ and hence there is no opportunity of arbitrage (for more details (Thao, 2006)). This is the advantage of our approximate approach and we will use this model for pricing the European call option instead of SDE (8).

Note that we can write:

$d B_{t}^{\varepsilon}=\alpha \varphi_{t}^{\varepsilon} d t+\varepsilon^{\alpha} d W_{t}$

where $\varphi_{\mathrm{t}}^{\varepsilon}=\int_{0}^{\mathrm{t}}(\mathrm{t}-\mathrm{u}+\varepsilon)^{1-\alpha} \mathrm{dW}_{\mathrm{u}}, \alpha=1 / 2-\mathrm{H}$ and $0<\alpha<$ 1/2 ((Thao, 2006), Lemma 2.1).

Substituting (16) into Eq. 11, we obtain:

$d v_{t}^{\varepsilon}=\left(\omega+\left(\alpha \xi \varphi_{t}^{\varepsilon}-\theta\right) v_{t}^{\varepsilon}\right) d t+\xi \varepsilon^{\alpha} v_{t}^{\varepsilon} d W_{t}$ 
Consider the SDE (10) and (17). Define a function $\mathrm{U}$ on $\mathfrak{R}^{2}$ as follows:

$\mathrm{U}\left(\mathrm{x}_{1}, \mathrm{x}_{2}\right)=\mathrm{e}^{-\mathrm{r}(\mathrm{T}-\mathrm{t})} \max \left(\exp \left(\mathrm{x}_{1}-\kappa\right), 0\right)$.

By virtue of Eq. 14:

$\mathrm{u}(\overrightarrow{\mathrm{x}}, \mathrm{t})=\mathrm{E}_{\mathrm{M}}\left[\mathrm{U}\left(\overrightarrow{\mathrm{X}}_{\mathrm{T}}\right) \mid \overrightarrow{\mathrm{X}}_{\mathrm{t}}=\overrightarrow{\mathrm{x}}\right]$

$=\mathrm{e}^{-\mathrm{r}(\mathrm{T}-\mathrm{t})} \mathrm{E}_{\mathrm{M}}\left[\max \left(\exp \left(\left(\mathrm{L}_{\mathrm{t}}^{\varepsilon}\right)-\kappa\right), 0\right) \mid \mathbf{L}_{\mathrm{t}}^{\varepsilon}=\ell^{\varepsilon}, \mathrm{v}_{\mathrm{t}}^{\varepsilon}=\mathrm{v}^{\varepsilon}\right]$

$:=\mathrm{C}\left(\ell^{\varepsilon}, \mathrm{v}^{\varepsilon}, \mathrm{t} ; \kappa, \mathrm{T}\right)$

satisfies the following PIDE:

$0=\frac{\partial \mathrm{C}}{\partial \mathrm{t}}+\mathrm{f}_{1} \frac{\partial \mathrm{C}}{\partial \ell^{\varepsilon}}+\mathrm{f}_{2} \frac{\partial \mathrm{C}}{\partial \mathrm{v}^{\varepsilon}}+\frac{1}{2} \mathrm{~g}_{1}^{2} \frac{\partial^{2} \mathrm{C}}{\left(\partial \ell^{\varepsilon}\right)^{2}}$

$+\rho g_{1} g_{2} \frac{\partial^{2} \mathrm{C}}{\partial \ell^{\varepsilon} \partial \mathrm{v}^{\varepsilon}}+\frac{1}{2} \mathrm{~g}_{2}^{2} \frac{\partial^{2} \mathrm{C}}{\partial\left(\mathrm{v}^{\varepsilon}\right)^{2}}-\mathrm{rC}$

$+\lambda \int_{\Re}\left[\mathrm{C}\left(\ell^{\varepsilon}+\mathrm{y}, \mathrm{v}^{\varepsilon}, \mathrm{t} ; \kappa, \mathrm{T}\right)-\mathrm{C}\left(\ell^{\varepsilon}, \mathrm{v}^{\varepsilon}, \mathrm{t} ; \kappa, \mathrm{T}\right)\right] \phi_{\mathrm{Y}}(\mathrm{y}) \mathrm{dy}$.

In the current state variable, the last line of Eq. 15 becomes:

$\mathrm{C}\left(\ell^{\varepsilon}, \mathrm{v}^{\varepsilon}, \mathrm{t} ; \kappa, \mathrm{T}\right)=\mathrm{e}^{\ell^{\varepsilon}} \mathrm{P}_{1}\left(\ell^{\varepsilon}, \mathrm{v}^{\varepsilon}, \mathrm{t} ; \kappa, \mathrm{T}\right)$

$-\mathrm{e}^{\kappa-\mathrm{r}(\mathrm{T}-\mathrm{t})} \mathrm{P}_{2}\left(\ell^{\varepsilon}, \mathrm{v}^{\varepsilon}, \mathrm{t} ; \kappa, \mathrm{T}\right)$.

The following lemma shows the relationship between $P_{1}$ and $P_{2}$ in the option value of the Eq. 19 .

Lemma 1: The probability $P_{1}$ in the option value of the Eq. 19 satisfies the following PIDE:

$$
\begin{aligned}
& 0=\frac{\partial \mathrm{P}_{1}}{\partial \mathrm{t}}+\mathrm{A}\left[\mathrm{P}_{1}\right]\left(\ell^{\varepsilon}, \mathrm{v}^{\varepsilon}, \mathrm{t} ; \kappa, \mathrm{T}\right)+\mathrm{v}^{\varepsilon} \frac{\partial \mathrm{P}_{1}}{\partial \ell^{\varepsilon}}+\rho \xi \varepsilon^{\alpha}\left(\mathrm{v}^{\varepsilon}\right)^{3 / 2} \frac{\partial \mathrm{P}_{1}}{\partial \mathrm{v}^{\varepsilon}} \\
& +\left(\mathrm{r}-\lambda \mathrm{E}_{\mathrm{M}}\left(\mathrm{Y}_{\mathrm{t}}\right)\right) \mathrm{P}_{1} \\
& +\lambda \int_{\Re}\left[\left(\mathrm{e}^{\mathrm{y}}-1\right) \mathrm{P}_{1}\left(\ell^{\varepsilon}+\mathrm{y}, \mathrm{v}^{\varepsilon}, \mathrm{t} ; \kappa, \mathrm{T}\right)\right] \phi_{\mathrm{Y}}(\mathrm{y}) \mathrm{dy} \\
& :=\frac{\partial \mathrm{p}_{1}}{\partial \mathrm{t}}+\mathrm{A}_{1}\left[\mathrm{P}_{1}\right]\left(\ell^{\varepsilon}, \mathrm{v}^{\varepsilon}, \mathrm{t} ; \kappa, \mathrm{T}\right)
\end{aligned}
$$

subject to the boundary condition at expiration time $\mathrm{t}=$ $\mathrm{T}$ :

$$
\mathrm{P}_{1}\left(\ell^{\varepsilon}, \mathrm{v}^{\varepsilon}, \mathrm{T} ; \kappa, \mathrm{T}\right)=1_{\ell^{\varepsilon}>\kappa^{*}}
$$

And the probability $\mathrm{P}_{2}$ in the option value of the Eq. 19 satisfies the following PIDE: $0=\frac{\partial \mathrm{P}_{2}}{\partial \mathrm{t}}\left(\ell^{\varepsilon}, \mathrm{v}^{\varepsilon}, \mathrm{t} ; \kappa, \mathrm{T}\right)+\mathrm{rP}_{2}$

$:=\frac{\partial \mathrm{P}_{2}}{\partial \mathrm{t}}+\mathrm{A}_{2}\left[\mathrm{P}_{2}\right]\left(\ell^{\varepsilon}, \mathrm{v}^{\varepsilon}, \mathrm{t} ; \kappa, \mathrm{T}\right)$

subject to the boundary condition at expiration time $\mathrm{t}=\mathrm{T}$ :

$\mathrm{P}_{2}\left(\ell^{\varepsilon}, \mathrm{v}^{\varepsilon}, \mathrm{T} ; \kappa, \mathrm{T}\right)=1_{\ell^{\varepsilon}>\kappa}$

Where:

$$
\begin{aligned}
& \mathrm{A}[\mathrm{f}]\left(\ell^{\varepsilon}, \mathrm{v}^{\varepsilon}, \mathrm{t} ; \kappa, \mathrm{T}\right):=\left(\mathrm{r}-\lambda \mathrm{E}\left[\mathrm{Y}_{\mathrm{t}}\right]-\frac{1}{2} \mathrm{v}^{\varepsilon}\right) \frac{\partial \mathrm{f}}{\partial \ell^{\varepsilon}} \\
& +\left(\omega+\left(\alpha \xi \varphi^{\varepsilon}-\theta\right) \mathrm{v}^{\varepsilon}\right) \frac{\partial \mathrm{f}}{\partial \mathrm{v}^{\varepsilon}}+\frac{1}{2} \mathrm{v}^{\varepsilon} \frac{\partial^{2} \mathrm{f}}{\partial\left(\ell^{\varepsilon}\right)^{2}} \\
& +\rho \xi \varepsilon^{\alpha}\left(\mathrm{v}^{\varepsilon}\right)^{3 / 2} \frac{\partial^{2} \mathrm{f}}{\partial \ell^{\varepsilon} \partial \mathrm{v}^{\varepsilon}}+\frac{1}{2} \xi^{2} \varepsilon^{2 \alpha}\left(\mathrm{v}^{\varepsilon}\right)^{2} \frac{\partial^{2} \mathrm{f}}{\partial\left(\mathrm{v}^{\varepsilon}\right)^{2}} \\
& -\mathrm{rf}+\lambda \int_{\Re}\left[\begin{array}{l}
\mathrm{f}\left(\ell^{\varepsilon}+\mathrm{y}, \mathrm{v}^{\varepsilon}, \mathrm{t} ; \kappa, \mathrm{T}\right) \\
-\mathrm{f}\left(\ell^{\varepsilon}, \mathrm{v}^{\varepsilon}, t ; \kappa, \mathrm{T}\right)
\end{array}\right] \phi_{\mathrm{Y}}(\mathrm{y}) \mathrm{dy}
\end{aligned}
$$

Note that $1_{\ell^{\varepsilon}>\kappa}=1$ if $\ell^{\varepsilon}>\kappa$ and otherwise $1_{\ell^{\varepsilon}>\kappa}=0$.

Proof: Calculating the partial derivatives of function $\mathrm{C}\left(\ell^{\varepsilon}, \mathrm{v}^{\varepsilon}, \mathrm{t} ; \kappa, \mathrm{T}\right)$ in Eq. 19 and substituting it's in Eq. 18 then separating it by assumed independent terms $\mathrm{P}_{1}$ and $\mathrm{P}_{2}$. This gives two PIDEs for the risk-neutralized probability $P_{j}\left(\ell^{\varepsilon}, v^{\varepsilon}, t ; \kappa, T\right), j=1,2$. For $j=1$ we have:

$$
\begin{aligned}
& 0=\frac{\partial \mathrm{P}_{1}}{\partial \mathrm{t}}+\left(\mathrm{r}-\lambda \mathrm{E}_{\mathrm{M}}\left(\mathrm{Y}_{\mathrm{t}}\right)-\frac{1}{2} \mathrm{v}^{\varepsilon}\right)\left(\frac{\partial \mathrm{P}_{1}}{\partial \ell^{\varepsilon}}+\mathrm{P}_{1}\right) \\
& +\left(\omega+\left(\alpha \xi \varphi_{\mathrm{t}}^{\varepsilon}-\theta\right) \mathrm{v}^{\varepsilon}\right) \frac{\partial \mathrm{P}_{1}}{\partial \mathrm{v}^{\varepsilon}}+\frac{1}{2} \mathrm{v}^{\varepsilon}\left(\frac{\partial^{2} \mathrm{P}_{1}}{\left(\ell^{\varepsilon}\right)^{2}}+2 \frac{\partial \mathrm{P}_{1}}{\partial \ell^{\varepsilon}}+\mathrm{P}_{1}\right) \\
& +\rho \xi \varepsilon^{\alpha}\left(\mathrm{v}^{\varepsilon}\right)^{3 / 2}\left(\frac{\partial^{2} \mathrm{P}_{1}}{\partial \ell^{\varepsilon} \partial \mathrm{v}^{\varepsilon}}+\frac{\partial \mathrm{P}_{1}}{\partial \mathrm{v}^{\varepsilon}}\right)+\frac{1}{2} \xi^{2} \varepsilon^{2 \alpha}\left(\mathrm{v}^{\varepsilon}\right)^{2} \frac{\partial^{2} \mathrm{P}_{1}}{\partial\left(\mathrm{v}^{\varepsilon}\right)^{2}}-\mathrm{rP}_{1} \\
& +\lambda \int_{\Re}\left[\begin{array}{c}
\left(\mathrm{e}^{\mathrm{y}}-1\right) \mathrm{P}_{1}\left(\ell^{\varepsilon}+\mathrm{y}, \mathrm{v}^{\varepsilon}, \mathrm{t} ; \mathrm{T}\right) \\
+\left(\mathrm{P}_{1}\left(\ell^{\varepsilon}+\mathrm{y}, \mathrm{v}^{\varepsilon}, \mathrm{t} ; \mathrm{T}\right)-\mathrm{P}_{1}\left(\ell^{\varepsilon}+\mathrm{y}, \mathrm{v}^{\varepsilon}, \mathrm{t} ; \mathrm{T}\right)\right.
\end{array}\right] \phi \mathrm{Y}(\mathrm{y}) \mathrm{dy}
\end{aligned}
$$

subject to the boundary condition at the expiration time $\mathrm{t}=\mathrm{T}$ according to Eq. 21. By using the notation in Eq. 24 to PIDE (25) we get Eq. 20:

For $\mathrm{P}_{2}\left(\ell^{\varepsilon}, \mathrm{v}^{\varepsilon}, \mathrm{t} ; \kappa, \mathrm{T}\right)$, we have:

$$
\begin{aligned}
& 0=\frac{\partial \mathrm{P}_{2}}{\partial \mathrm{t}}+\mathrm{rP}_{2}+\left(\mathrm{r}-\lambda \mathrm{E}_{\mathrm{M}}\left(\mathrm{Y}_{\mathrm{t}}\right)-\frac{1}{2} \mathrm{v}^{\varepsilon}\right)\left(\frac{\partial \mathrm{P}_{2}}{\partial \ell^{\varepsilon}}\right) \\
& +\left(\omega\left(\alpha \xi \varphi_{\mathrm{t}}^{\varepsilon}-\theta\right) \mathrm{v}^{\varepsilon}\right) \frac{\partial \mathrm{P}_{2}}{\partial \mathrm{v}^{\varepsilon}}+\frac{1}{2} \mathrm{v}^{\varepsilon} \frac{\partial^{2} \mathrm{P}_{2}}{\partial\left(\ell^{\varepsilon}\right)^{2}} \\
& +\rho \xi \varepsilon^{\alpha}\left(\mathrm{v}^{\varepsilon}\right)^{3 / 2} \frac{\partial^{2} \mathrm{P}_{1}}{\partial \ell^{\varepsilon} \partial \mathrm{v}^{\varepsilon}}+\frac{1}{2} \xi^{2} \varepsilon^{2 \alpha}\left(\mathrm{v}^{\varepsilon}\right)^{2} \frac{\partial^{2} \mathrm{P}_{1}}{\partial\left(\mathrm{v}^{\varepsilon}\right)^{2}} \\
& -\mathrm{rP}_{2}+\lambda \int_{\Re}\left[\begin{array}{l}
\mathrm{P}_{2}\left(\ell^{\varepsilon}+\mathrm{y}, \mathrm{v}^{\varepsilon}, \mathrm{t} ; \kappa, \mathrm{T}\right) \\
-\mathrm{P}_{2}\left(\ell^{\varepsilon}+\mathrm{y}, \mathrm{v}^{\varepsilon}, \mathrm{t} ; \kappa, \mathrm{T}\right)
\end{array}\right] \phi_{\mathrm{Y}}(\mathrm{y}) \mathrm{dy}
\end{aligned}
$$


subject to the boundary condition at expiration time $\mathrm{t}=$ $\mathrm{T}$ according to Eq. 23. Again, by using the notation (24) to PIDE (26) we get Eq. 22. The proof is now completed.

Next, an approximate formula of European call option is calculated. For $\mathrm{j}=1,2$ the characteristic functions for $\mathrm{P}_{\mathrm{j}}\left(\ell^{\varepsilon}, \mathrm{v}^{\varepsilon}, \mathrm{t} ; \kappa, \mathrm{T}\right)$ with respect to the variable $\mathrm{k}$ are defined by:

$f_{j}\left(\ell^{\varepsilon}, v^{\varepsilon}, t: x, T\right):=-\int_{-\infty}^{\infty} e^{i x \kappa} d P_{j}\left(\ell^{\varepsilon}, v^{\varepsilon}, t: \kappa, T\right)$

with a minus sign to account for the negativity of the measure $\mathrm{dP}_{\mathrm{j}}$. Note that $\mathrm{f}_{\mathrm{j}}$ also satisfies similar PIDEs:

$\frac{\partial f_{j}}{\partial t}+A_{j}\left[f_{j}\right]\left(\ell^{\varepsilon}, v^{\varepsilon}, t ; \kappa, T\right)=0$

with the respective boundary conditions:

$$
\begin{aligned}
& \mathrm{f}_{\mathrm{j}}\left(\ell^{\varepsilon}, \mathrm{v}^{\varepsilon}, \mathrm{T} ; \mathrm{x}, \mathrm{T}\right)=-\int_{-\infty}^{\infty} \mathrm{e}^{\mathrm{j} x \mathrm{k}} \mathrm{dP} \mathrm{P}_{\mathrm{j}}\left(\ell^{\varepsilon}, \mathrm{v}^{\varepsilon}, \mathrm{T} ; \kappa, \mathrm{T}\right) \\
& =-\int_{-\infty}^{\infty} \mathrm{e}^{\mathrm{ix \kappa}}\left(-\delta\left(\ell^{\varepsilon}-\kappa\right) \mathrm{d} \kappa\right)=\mathrm{e}^{\mathrm{i} x \ell^{\varepsilon}}
\end{aligned}
$$

Since:

$\mathrm{dP}_{\mathrm{j}}\left(\ell^{\varepsilon}, \mathrm{v}^{\varepsilon}, \mathrm{T}: \kappa, \mathrm{T}\right)=\mathrm{d} 1_{\ell^{\varepsilon}>\mathrm{\kappa}}=\mathrm{dH}\left(\ell^{\varepsilon}-\kappa\right)=-\delta\left(\ell^{\varepsilon}-\kappa\right) \mathrm{d} \kappa$

Note that the probabilities $P_{j}, j=1,2$ are the conditional probabilities that the option expires in-themoney that is:

$P_{j}=M\left\{L_{T}^{\varepsilon} \geq \log K \mid L_{t}^{\varepsilon}=\ell^{\varepsilon}, v_{t}^{\varepsilon}=v\right\}$

where again $\mathrm{L}_{\mathrm{t}}^{\varepsilon}=\log \mathrm{S}_{\mathrm{t}}^{\varepsilon}$ and $\left(\mathrm{S}_{\mathrm{t}}^{\varepsilon}, \mathrm{v}_{\mathrm{t}}^{\varepsilon}\right)$ evolves according to Eq. 10 and 11 respectively.

Using a Fourier transform method one gets:

$\mathrm{dP}_{\mathrm{j}}\left(\ell^{\varepsilon}, \mathrm{v}^{\varepsilon}, \mathrm{t}: \kappa, \mathrm{T}\right)=\frac{1}{2}+\frac{1}{\pi} \int_{0^{+}}^{+\infty} \operatorname{Re}\left[\frac{\mathrm{e}^{-\mathrm{ix \kappa}} \mathrm{f}_{\mathrm{j}}\left(\ell^{\varepsilon}, \mathrm{v}^{\varepsilon}, \mathrm{t}: \mathrm{x}, \mathrm{T}\right)}{\mathrm{ix}}\right] \mathrm{dx}$

where, $\mathrm{j}=1,2$ and the characteristic function $\mathrm{f}_{\mathrm{j}}\left(\ell^{\varepsilon}, \mathrm{v}^{\varepsilon}, \mathrm{t} ; \mathrm{x}, \mathrm{T}\right)$ also satisfy the PIDEs in lemma 1 , namely Eq. 20 and 22 and $\operatorname{Re}[$.$] denoting the real$ component of a complex number. The practice to solving of this kind of equations is to guess the general form of the solution. The following lemma shows how to calculate the probabilities $\mathrm{P}_{1}$ and $\mathrm{P}_{2}$ as they appeared in Lemma 1.

Lemma 2: The probabilities $\mathrm{P}_{1}$ and $\mathrm{P}_{2}$ can be calculated by Eq. 28 where the explicit expressions of the characteristic functions is given as follows. (i) The characteristic function $\mathrm{f}_{1}$ is given by:

$\mathrm{f}_{\mathrm{j}}\left(\ell^{\varepsilon}, \mathrm{v}^{\varepsilon}, \mathrm{t} ; \mathrm{x}, \mathrm{t}+\tau\right)=\exp \left(\mathrm{g}_{1}(\tau)+\mathrm{v}^{\varepsilon} \mathrm{h}_{1}(\tau)+\mathrm{jx} \ell^{\varepsilon}\right)$

where, $\tau=\mathrm{T}-\mathrm{t}$ :

$$
\begin{aligned}
& \mathrm{g}_{1}(\tau)=\left[\mathrm{r}-\lambda \mathrm{E}_{\mathrm{M}}\left(\mathrm{Y}_{\mathrm{t}}\right) \mathrm{jx}-\lambda \mathrm{E}_{\mathrm{M}}\left(\mathrm{Y}_{\mathrm{t}}\right)\right] \tau \\
& +\tau \lambda \int_{\Re}\left(\mathrm{e}^{(\mathrm{ix}+1) \mathrm{y}}-1\right) \phi_{\mathrm{Y}}(\mathrm{y}) \mathrm{dy} \\
& -\frac{2 \omega}{\xi^{2} \varepsilon^{2 \alpha} \mathrm{v}^{\varepsilon}}\left[\log \left(1-\frac{\left(\Delta_{1}+\eta_{1}\right)+\left(1-\mathrm{e}^{\Delta_{1} \tau}\right)}{2 \Delta_{1}}\right)+\left(\Delta_{1}+\eta_{1}\right) \tau\right] \\
& \mathrm{h}_{1}(\tau)=\frac{\left(\eta_{1}^{2}-\Delta_{1}^{2}\right)\left(\mathrm{e}^{\Delta_{1} \tau}-1\right)}{\xi^{2} \varepsilon^{2 \alpha \varepsilon} \mathrm{v}^{\varepsilon}\left(\eta_{1}+\Delta_{1}-\left(\eta_{1}-\Delta_{1}\right) \mathrm{e}^{\Delta_{1} \tau}\right)}, \\
& \eta_{1}=\rho \xi \varepsilon^{\alpha} \sqrt{\mathrm{v}^{\varepsilon}}(1+\mathrm{ix})+\left(\alpha \xi \ell_{\mathrm{t}}^{\varepsilon}-\theta\right) \\
& \text { and } \Delta_{1}=\sqrt{\eta_{1}^{2}-\xi^{2} \varepsilon^{2 \alpha} \mathrm{ix}(\mathrm{ix}+1)}
\end{aligned}
$$

(ii) The characteristic function $\mathrm{f}_{2}$ is given by:

$$
f_{2}\left(\ell^{\varepsilon}, v^{\varepsilon}, t ; x, t+\tau\right)=\exp \left(g_{2}(\tau)+v^{\varepsilon} h_{2}(\tau)+i x \ell^{\varepsilon}+r \tau\right)
$$

Where:

$$
\begin{aligned}
& \mathrm{g}_{2}(\tau)=\left[\mathrm{r}-\lambda \mathrm{E}_{\mathrm{M}}\left[\mathrm{Y}_{\mathrm{t}}\right] \mathrm{iy}-\mathrm{r}\right] \tau+\tau \lambda \int_{\Re}\left(\mathrm{e}^{\mathrm{ixy}}-1\right) \phi_{\mathrm{Y}}(\mathrm{y}) \mathrm{dy} \\
& -\frac{2 \omega}{\xi^{2} \varepsilon^{2 \alpha} \mathrm{v}^{\varepsilon}}\left[\log \left(1-\frac{\left(\Delta_{2}+\eta_{2}\right)+\left(1-\mathrm{e}^{\Delta_{2} \tau}\right)}{2 \Delta_{2}}\right)+\left(\Delta_{2}+\eta_{2}\right) \tau\right] \\
& \mathrm{h}_{2}(\tau)=\frac{\left(\eta_{2}^{2}-\Delta_{2}^{2}\right)\left(\mathrm{e}^{\Delta_{2} \tau}-1\right)}{\xi^{2} \varepsilon^{2 \alpha} \mathrm{v}^{\varepsilon}\left(\eta_{2}+\Delta_{2}-\left(\eta_{2}-\Delta_{2}\right) \mathrm{e}^{\Delta_{2} \tau}\right)} \\
& \eta_{2}=\rho \xi \varepsilon^{\alpha} \sqrt{\mathrm{v}^{\varepsilon}} \mathrm{ix}+\left(\alpha \xi \varphi_{\mathrm{t}}^{\varepsilon}-\theta\right) \\
& \text { and } \Delta_{2}=\sqrt{\eta_{2}^{2}+\xi^{2} \varepsilon^{2 \alpha} \mathrm{v}^{\varepsilon} \mathrm{ix}(\mathrm{ix}-1)}
\end{aligned}
$$

Proof: Proof of (i). To solve for the characteristic explicitly, letting $\tau=\mathrm{T}-\mathrm{t}$ be the time-to-go. Following (Heston, 1993), we conjecture that the function $f_{1}$ is given by:

$$
f_{1}\left(\ell^{\varepsilon}, v^{\varepsilon}, t ; x, t+\tau\right)=\exp \left(\left(g_{1}\right)(\tau)+v^{\varepsilon} h_{1}(\tau)+i x \ell^{\varepsilon}\right)
$$

and the boundary condition $\mathrm{g}_{1}(0)=0=\mathrm{h}_{1}(0)$. This conjecture exploits the linearity of the coefficient in PIDE (27). 
Note that the characteristic functions of $f_{1}$ always exists. In order to substitute (29) into (27), firstly, we calculate the partial derivative of $f_{1}$ and substitute it's into Eq. 27. After canceling the common factor of $f_{1}$, we get a simplified form as follows:

$$
\begin{aligned}
& 0=\mathrm{g}_{1}^{\prime}(\tau)-\mathrm{v}^{\varepsilon} \mathrm{h}_{1}^{\prime}(\tau)+\left(\mathrm{r}-\lambda \mathrm{E}_{\mathrm{M}}\left[\mathrm{Y}_{\mathrm{t}}\right]+\frac{1}{2} \mathrm{v}^{\varepsilon}\right) \mathrm{ix} \\
& +\left(\omega+\left(\alpha \xi \varphi_{\mathrm{t}}^{\varepsilon}-\theta\right) \mathrm{v}^{\varepsilon}\right)+\rho \xi \varepsilon^{\alpha}\left(\mathrm{v}^{\varepsilon}\right)^{3 / 2} \mathrm{~h}_{1}(\tau) \\
& -\frac{1}{2} \mathrm{v}^{\varepsilon} \mathrm{x}^{2}+\rho \xi \varepsilon^{\alpha}\left(\mathrm{v}^{\varepsilon}\right)^{3 / 2} \mathrm{ixh}_{1}(\tau)+\frac{1}{2} \xi^{2} \varepsilon^{2 \alpha}\left(\mathrm{v}^{\varepsilon}\right)^{2} \mathrm{~h}_{1}^{2}(\tau) \\
& -\lambda \mathrm{E}_{\mathrm{M}}\left[\mathrm{Y}_{\mathrm{t}}\right]+\lambda \int_{\Re}\left(\mathrm{e}^{(\mathrm{ix}+1) \mathrm{y}}-1\right) \phi_{\mathrm{Y}}(\mathrm{y}) \mathrm{dy}
\end{aligned}
$$

By separating the order $\mathrm{v}^{\varepsilon}$ and ordering the remaining terms, we can reduce it to two Ordinary Differential Equations (ODEs):

$$
\begin{aligned}
& \mathrm{h}_{1}^{\prime}(\tau)=\frac{1}{2} \xi^{2} \varepsilon^{2 \alpha} \mathrm{v}^{\varepsilon} \mathrm{h}_{1}^{2}(\tau)+\left(\rho \xi \varepsilon^{\alpha} \sqrt{\mathrm{v}^{\varepsilon}}(1+\mathrm{ix})\right. \\
& \left.+\left(\alpha \xi \varphi_{\mathrm{t}}^{\varepsilon}-\theta\right)\right) \mathrm{h}_{1}(\tau)+\frac{1}{2} \mathrm{ix}-\frac{1}{2} \mathrm{x}^{2} \\
& \mathrm{~g}_{1}^{\prime}(\tau)=\omega \mathrm{h}_{1}(\tau)+\left(\mathrm{r}-\lambda \mathrm{E}_{\mathrm{M}}\left[\mathrm{Y}_{\mathrm{t}}\right]\right) \mathrm{ix}-\lambda \mathrm{E}_{\mathrm{M}}\left[\mathrm{Y}_{\mathrm{t}}\right] \\
& +\lambda \int_{\Re}\left(\mathrm{e}^{(\mathrm{ix}+1) \mathrm{y}}-1\right) \phi_{\mathrm{Y}}(\mathrm{y}) \mathrm{dy}
\end{aligned}
$$

Let $\eta_{1}=\rho \xi \varepsilon^{\alpha} \sqrt{v^{\varepsilon}}(1+i x)+\left(\alpha \xi \varphi_{t}^{\varepsilon}-\theta\right)$ and substitute it to Eq. 30.

We get:

$$
\begin{aligned}
& \mathrm{h}_{1}^{\prime}(\tau)=\frac{1}{2} \xi^{2} \varepsilon^{2 \alpha} \mathrm{v}^{\varepsilon}\left(\mathrm{h}_{1}^{2}(\tau)+\frac{2 \eta_{1}}{\xi^{2} \varepsilon^{2 \alpha} \mathrm{v}^{\varepsilon}} \mathrm{h}_{1}(\tau)+\frac{1}{\xi^{2} \varepsilon^{2 \alpha} \mathrm{v}^{\varepsilon}} \mathrm{ix}(\mathrm{ix}+1)\right) \\
& =\frac{1}{2} \xi^{2} \varepsilon^{2 \alpha}\left(\mathrm{h}_{1}(\tau)+\frac{2 \eta_{1}+\sqrt{4 \eta_{1}^{2}-4 \xi^{2} \varepsilon^{2 \alpha} \mathrm{v}^{\varepsilon} \mathrm{ix}(\mathrm{ix}+1)}}{2 \xi^{2} \varepsilon^{2 \alpha} \mathrm{v}^{\varepsilon}}\right) \\
& \times\left(\mathrm{h}_{1}(\tau)+\frac{2 \eta_{1}-\sqrt{4 \eta_{1}^{2}-4 \xi^{2} \varepsilon^{2 \alpha} \mathrm{v}^{\varepsilon} \mathrm{ix}(\mathrm{ix}+1)}}{2 \xi^{2} \varepsilon^{2 \alpha} \mathrm{v}^{\varepsilon}}\right) \\
& =\frac{1}{2} \xi^{2} \varepsilon^{2 \alpha} \mathrm{v}^{\varepsilon}\left(\mathrm{h}_{1}(\tau)+\frac{\eta_{1}+\Delta_{1}}{\xi^{2} \varepsilon^{2 \alpha} \mathrm{v}^{\varepsilon}}\right)\left(\mathrm{h}_{1}(\tau) \frac{\eta_{1}-\Delta_{1}}{\xi^{2} \varepsilon^{2 \alpha} \mathrm{v}^{\varepsilon}}\right)
\end{aligned}
$$

Where:

$$
\Delta_{1}=\sqrt{\eta_{1}^{2}-\xi^{2} \varepsilon^{2 \alpha} v^{\varepsilon} \mathrm{ix}(\mathrm{ix}+1)}
$$

By method of variable separation, we have:

$$
\frac{2 \mathrm{dh}_{1}(\tau)}{\left(\mathrm{h}_{1}(\tau)+\frac{\eta_{1}+\Delta_{1}}{\xi^{2} \varepsilon^{2 \alpha} \mathrm{v}^{\varepsilon}}\right)\left(\mathrm{h}_{1}(\tau)+\frac{\eta_{1}-\Delta_{1}}{\xi^{2} \varepsilon^{2 \alpha} \mathrm{v}^{\varepsilon}}\right)}=\xi^{2} \varepsilon^{2 \alpha} \mathrm{v}^{\varepsilon} \mathrm{d} \tau
$$

Using partial fractions, we get:

$\frac{1}{\Delta_{1}}\left(\frac{1}{h_{1}(\tau)+\frac{\eta_{1}-\Delta_{1}}{\xi^{2} \varepsilon^{2 \alpha} v^{\varepsilon}}}-\frac{1}{h_{1}(\tau)+\frac{\eta_{1}+\Delta_{1}}{\xi^{2} \varepsilon^{2 \alpha} v^{\varepsilon}}}\right) \mathrm{dh}_{1}(\tau)=\mathrm{d} \tau$

Integrating both sides, we obtain:

$$
\log \left(\frac{h_{1}(\tau)+\frac{\eta_{1}-\Delta_{1}}{\xi^{2 \varepsilon} \alpha v^{\varepsilon}}}{h_{1}(\tau)+\frac{\eta_{1}+\Delta_{1}}{\xi^{2 \varepsilon} \alpha v^{\varepsilon}}}\right)=\Delta_{1} \tau+C
$$

Using boundary condition $h_{1}(\tau=0)=0$ we get:

$$
C=\log \left(\frac{\eta_{1}-\Delta_{1}}{\eta_{1}+\Delta_{1}}\right)
$$

Solving for $\mathrm{h}_{1}$, we obtain:

$$
\mathrm{h}_{1}(\tau)=\frac{\left(\eta_{1}^{2}-\Delta_{1}^{2}\right)\left(\mathrm{e}^{\Delta_{1} \tau}-1\right)}{\xi^{2} \varepsilon^{2 \alpha} \mathrm{c}^{\varepsilon}\left(\eta_{1}+\Delta_{1}-\left(\eta_{1}-\Delta_{1}\right) \mathrm{e}^{\Delta_{1} \tau}\right)}
$$

In order to solve $g_{1}(\tau)$ explicitly, we substitute $h_{1}$ into Eq. 31 and integrate with respect to $\mathrm{T}$ on both sides.

Then we get:

$$
\begin{aligned}
& \mathrm{g}_{1}(\tau)=\left[\left(\mathrm{r}-\lambda \mathrm{E}_{\mathrm{M}}\left(\mathrm{Y}_{\mathrm{t}}\right)\right) \mathrm{ix}-\lambda \mathrm{E}\left(\mathrm{Y}_{\mathrm{t}}\right)\right] \tau \\
& +\tau \lambda \int_{\Re}\left(\mathrm{e}^{(\mathrm{ix}+1) \mathrm{y}}-1\right) \phi_{\mathrm{Y}}(\mathrm{y}) \mathrm{dy} \\
& -\frac{2 \omega}{\xi^{2} \varepsilon^{2 \alpha} \mathrm{v}^{\varepsilon}}\left[\log \left(1-\frac{\left(\Delta_{1}+\eta_{1}\right)+\left(1-\mathrm{e}^{\Delta_{1} \tau}\right)}{}\right)+\left(\Delta_{1}+\eta_{1}\right) \tau\right]
\end{aligned}
$$

Proof of (ii). The details of the proof are similar to case (i). Hence, we have:

$f_{2}\left(\ell^{\varepsilon}, v^{\varepsilon}, t ; y, t+\tau\right)=\exp \left(g_{2}(\tau)+v^{\varepsilon} h_{2}(\tau)+i y \ell^{\varepsilon}+r \tau\right)$

where, $\mathrm{g}_{2}(\tau), \mathrm{h}_{2}(\tau), \eta_{2}$ and $\Delta_{2}$ are as given in the Lemma.

We can thus evaluate the characteristic functions in explicit form. However, we are interested in the riskneutral probabilities $P_{j}$. These can be inverted from the characteristic functions by performing the following integration: 


$$
\begin{aligned}
& \hat{P}_{j}\left(S_{t}^{\varepsilon}, v_{t}^{\varepsilon} ; K, T\right)=P_{j}\left(\ell^{\varepsilon}, v^{\varepsilon}, t ; \kappa, T\right) \\
& =\frac{1}{2}+\frac{1}{\pi} \int_{0+}^{+\infty} \operatorname{Re}\left[\frac{e^{-i x \kappa} f_{j}\left(\ell^{\varepsilon}, v_{t}^{\varepsilon} t ; x, T\right)}{i x}\right] d x
\end{aligned}
$$

for $\mathrm{j}=1,2$, where $\ell^{\varepsilon}=\log \left(S_{\mathrm{t}}^{\varepsilon}\right), \mathrm{v}^{\varepsilon}=\log \left(\mathrm{v}_{\mathrm{t}}^{\varepsilon}\right)$, and $\kappa=\log (\mathrm{K})$.

To verify the above equation, firstly we note that:

$$
\begin{aligned}
& E_{M}\left[e^{i x\left(\log \left(S_{t}^{\varepsilon}-\log (K)\right)\right.} \mid \log \left(S_{t}^{\varepsilon}\right)=L_{t}^{\varepsilon}, v_{t}^{\varepsilon}=v^{\varepsilon}\right] \\
& =E_{M}\left[e^{i x\left(\ell^{\varepsilon}-K\right)} \mid L_{t}^{\varepsilon}=\ell^{\varepsilon}, v_{t}^{\varepsilon}=v^{\varepsilon}\right]
\end{aligned}
$$

The computation of the right of above equation are:

$$
\begin{aligned}
& \int_{-\infty}^{+\infty} \mathrm{e}^{-\mathrm{ix}\left(\ell^{\varepsilon}-\kappa\right)} \mathrm{dP}_{\mathrm{j}}\left(\ell^{\varepsilon}, \mathrm{v}^{\varepsilon}, \mathrm{t} ; \kappa, \mathrm{T}\right)=\mathrm{e}^{-\mathrm{i} x \kappa} \int_{-\infty}^{+\infty} \mathrm{e}^{-\mathrm{ix} \ell^{\varepsilon}} \mathrm{dP}_{\mathrm{j}}\left(\ell^{\varepsilon}, \mathrm{v}^{\varepsilon}, \mathrm{t} ; \kappa, \mathrm{T}\right) \\
& =\mathrm{e}^{-\mathrm{ix} \kappa} \int_{-\infty}^{+\infty} \mathrm{e}^{\mathrm{i} x \ell^{\varepsilon}}\left(-\delta\left(\ell^{\varepsilon}-\kappa\right) \mathrm{d} \kappa\right)=\mathrm{e}^{-\mathrm{ix \kappa}} \mathrm{f}_{\mathrm{j}}\left(\ell^{\varepsilon}, \varepsilon, \mathrm{t} ; \mathrm{x}, \mathrm{T}\right)
\end{aligned}
$$

Then:

$$
\begin{aligned}
& \frac{1}{2}+\frac{1}{\pi} \int_{0+}^{+\infty} \operatorname{Re}\left[\frac{\mathrm{e}^{-\mathrm{i} \infty \mathrm{x}} \mathrm{f}_{\mathrm{j}}\left(\ell^{\varepsilon}, \mathrm{v}_{\mathrm{t}}^{\varepsilon} \mathrm{t} ; \mathrm{x}, \mathrm{T}\right)}{\mathrm{ix}}\right] \mathrm{dx} \\
& =\frac{1}{2}+\frac{1}{\pi} \int_{0+}^{+\infty} \operatorname{Re}\left[\frac{\mathrm{E}_{\mathrm{M}}\left[\mathrm{e}^{\mathrm{ix}\left(\log \left(\mathrm{S}_{\mathrm{t}}^{\varepsilon}\right)-\log (\mathrm{\kappa})\right)} \mid \log \left(\mathrm{S}_{\mathrm{t}}^{\varepsilon}\right)=\mathrm{L}_{\mathrm{t}}^{\varepsilon}, \mathrm{v}_{\mathrm{t}}^{\varepsilon}=\mathrm{v}^{\varepsilon}\right]}{\mathrm{ix}} \mathrm{dx}\right] \\
& =\mathrm{E}_{\mathrm{M}}\left[\frac{1}{2}+\frac{1}{\pi} \int_{0+}^{+\infty} \operatorname{Re}\left[\frac{\mathrm{e}^{\mathrm{ix}\left(\ell^{\varepsilon}-\kappa\right)}}{\mathrm{ix}}\right] \mathrm{dx} \mid \mathrm{L}_{\mathrm{t}}^{\varepsilon}=\ell^{\varepsilon}, \mathrm{v}_{\mathrm{t}}^{\varepsilon}=\mathrm{v}^{\varepsilon}\right] \\
& =\mathrm{E}_{\mathrm{M}}\left[\frac{1}{2}+\frac{1}{\pi} \int_{0+}^{+\infty} \frac{\sin \mathrm{x}\left(\ell^{\varepsilon}-\kappa\right)}{\mathrm{x}} \mathrm{dx} \mid \mathrm{L}_{\mathrm{t}}^{\varepsilon}=\ell^{\varepsilon}, \mathrm{v}_{\mathrm{t}}^{\varepsilon}=\mathrm{v}^{\varepsilon}\right] \\
& =\mathrm{E}_{\mathrm{M}}\left[\frac{1}{2}+\operatorname{sgn}\left(\kappa^{\varepsilon}-\kappa\right) \frac{1}{\pi} \int_{0+}^{+\infty} \frac{\sin (\mathrm{x})}{\mathrm{x}} \mathrm{dx} \mid \mathrm{L}_{\mathrm{t}}^{\varepsilon}=\ell^{\varepsilon}, \mathrm{v}_{\mathrm{t}}^{\varepsilon}=\mathrm{v}^{\varepsilon}\right] \\
& =\mathrm{E}_{\mathrm{M}}\left[\frac{1}{2}+\operatorname{sgn}\left(\ell^{\varepsilon}-\kappa\right) \mid \mathrm{L}_{\mathrm{t}}^{\varepsilon}=\mathrm{v}^{\varepsilon}, \mathrm{v}_{\mathrm{t}}^{\varepsilon}=\mathrm{v}^{\varepsilon}\right] \\
& =\mathrm{E}_{\mathrm{M}}\left[1_{\ell^{\varepsilon} \geq \mathrm{\kappa}} \mid \mathrm{L}_{\mathrm{t}}^{\varepsilon}=\ell^{\varepsilon}, \mathrm{v}_{\mathrm{t}}^{\varepsilon}=\mathrm{v}^{\varepsilon}\right]
\end{aligned}
$$

where we have used the Dirichlet formula $\int_{-\infty}^{+\infty} \frac{\sin (x)}{x} d x=1$ and the sgn function is defined as sgn $\operatorname{sgn}(x)=1$ if $x>0,0$ if $x=0$ and and -1 if $x<0$.

In summary, we have just proved the following main theorem.

Theorem 3: For each $\varepsilon>0$; the value of a European call option written on the model (10) and (11) is:

$$
\begin{aligned}
& \hat{\mathrm{C}}\left(\mathrm{S}_{\mathrm{t}}^{\varepsilon}, \mathrm{v}_{\mathrm{t}}^{\varepsilon}, \mathrm{t}, \mathrm{K}, \mathrm{T}\right)=\mathrm{S}_{\mathrm{t}}^{\varepsilon} \hat{\mathrm{P}}_{1}\left(\mathrm{~S}_{\mathrm{t}}^{\varepsilon}, \mathrm{v}_{\mathrm{t}}^{\varepsilon}, \mathrm{t}, \mathrm{K}, \mathrm{T}\right) \\
& -\mathrm{Ke}^{-\mathrm{r}(\mathrm{T}-\mathrm{t})} \hat{\mathrm{P}}_{2}\left(\mathrm{~S}_{\mathrm{t}}^{\varepsilon}, \mathrm{v}_{\mathrm{t}}^{\varepsilon}, \mathrm{t}, \mathrm{K}, \mathrm{T}\right)
\end{aligned}
$$

where, $\mathrm{P}_{1}$ and $\mathrm{P}_{2}$ are as given in Lemma 2 .

\section{DISCUSSION}

A simple and efficient numerical scheme for determining the approximate process $S_{t}^{\varepsilon}$ and $v_{t}^{\varepsilon}$ is presented.

In order to compute the value of $\hat{\mathrm{C}}\left(\mathrm{S}_{\mathrm{t}}^{\varepsilon}, \mathrm{v}_{\mathrm{t}}^{\varepsilon}, \mathrm{t} ; \mathrm{K}, \mathrm{T}\right)$ according to the formula as given in Theorem 3 , we firstly choose a real number $\varepsilon>0$, the solution that we get is the value of a European call option of the approximation model (10) with (11) and this value can be used as an approximating value of a call option of the fractional model (7) including model (8) as $\varepsilon$ approaches zero. As the Monte-Carlo based technique, it will generate discrete sample values $S_{i}^{\varepsilon}$ and $v_{i}^{\varepsilon}$ of the stock and its variance respectively, by discretizing the associated SDEs (10) and (11). A natural choice for this purpose is the Euler scheme:

$$
\begin{aligned}
& \hat{\mathrm{S}}_{\mathrm{i}+1}^{\varepsilon}=\mathrm{S}_{\mathrm{i}+1}^{\varepsilon}\left(\left(\mathrm{r}-\lambda \mathrm{E}_{\mathrm{M}}\left[\hat{\mathrm{Y}}_{\mathrm{i}}\right]\right) \mathrm{dt}+\sqrt{\hat{\mathrm{v}}_{\mathrm{i}}^{\varepsilon}} \Delta \mathrm{W}_{\mathrm{t}}\right)+\mathrm{S}_{\mathrm{i}-}^{\varepsilon} \mathrm{Y}_{\mathrm{i}} \Delta \mathrm{N}_{\mathrm{i}} \\
& \hat{\mathrm{V}}_{\mathrm{i}+1}^{\varepsilon}=\left(\omega-\theta \hat{\mathrm{v}}_{\mathrm{i}}^{\varepsilon}\right) \mathrm{h}+\xi \hat{\mathrm{v}}_{\mathrm{i}}^{\varepsilon} \Delta \mathrm{B}_{\mathrm{i}}^{\varepsilon}
\end{aligned}
$$

Where:

$\Delta \mathrm{W}_{\mathrm{t}}=$ Standard normal random variable with variance $\mathrm{h}$, which is defined as the time mesh-size $\Delta \mathrm{N}_{\mathrm{i}}=\mathrm{A}$ Poisson process with intensity $\lambda \mathrm{h}$

These processes, $\mathrm{W}$ and $\mathrm{N}$ are assumed independent. However, (Glasserman, 2004) suggests that the second-order scheme has a better convergence (less bias) for option pricing applications but this scheme quite complex. For the simulation of Brownian motion there are numerous procedures see (Glasserman, 2004). For a sample path of fractional Brownian motion in Eq. 10, we can be simulated, for fixed $t>0$, as:

$$
\begin{aligned}
& B_{t} \simeq \sum_{k=1}^{N}\left(t-\frac{k}{N} t\right)^{\alpha}\left[W_{(k+1)_{\frac{t}{N}}}-W_{k_{\frac{t}{N}}}\right] \\
& =\sum_{k=1}^{N}\left(t-\frac{k}{N} t\right)^{\alpha} \sqrt{\frac{t}{N}}\left[W_{(k+1)}-W_{k}\right] \\
& =\sqrt{\frac{t}{N}} \sum_{k=1}^{N}\left(t-\frac{k}{N} t\right)^{\alpha} g_{k}
\end{aligned}
$$

where, $\mathrm{g}_{\mathrm{k}} \sim \mathrm{N}(0,1)$ and $0<\alpha<1 / 2$. 
There are two basic estimation of the volatility process of Eq. 32 in the cast the volatility process is constant. The first method considers the function of density of transition from solution of Eq. 32. The second method proposes the estimate of the parameters of the model via the observation. Khaled and Samia (2010) for more details). In our case, the volatility of Eq. 32 is the stochastic process. There are many articles provided the estimation procedure for example see (Fiorentini et al., 2002).

\section{CONCLUSION}

An alternative fractional stochastic volatility model with jump is proposed in this study which the stock prices follows a geometric Brownian motion combining a compound Poisson processes and a stochastic volatility perturbed by a fractional Brownian motion. This proposed model exhibits a long memory of a stochastic volatility model that is not expressed in the classical stochastic volatility model. By using a fundamental result of the $\mathrm{L}^{2}$-approximation of a fractional Brownian motion, we provide an approximate solution of bivariate diffusion model. A relationship between stochastic differential equations and partial differential equations for a bivariate model is presented. The risk-neutral method for valuation of options are reviewed. By using the technique base on the characteristic function of an underlying assets, an approximate formula of a European options is derived in an explicit formula. Finally a numerical integration technique to simulation the fractional stochastic volatility are present.

\section{ACKNOWLEDGMENT}

This research is (partially) supported by Thailand Research Fun.

\section{REFERENCES}

Cheong, C.W., 2008. Volatility in Malaysian stock market: An empirical study using fractionally integrated Am. J. of Applied Sci. 5: 683-688. DOI: 10.3844/ajassp.2008.683.688
Cont, R. and P. Tankov, 2009. Financial Modeling with Jump Processes. 2nd Edn., Taylor and Francis, ISBN: 1420082191, 978-1420082197, pp: 606.

Fiorentini, G., A. Leon and G. Rubio, 2002. Estimation and empirical performance of Heston's stochastic volatility model: The case of a thinly traded market. J. Empirical Finance, 9: 225-255. DOI: 10.1016/S0927-5398(01)00052-4

Glasserman, P., 2004. Monte-Carlo in Financial Engineering. 1st Edn., Springer, ISBN: 0387004513, 978-0387004518, pp: 596.

Hanson, F.B., 2007. Applied Stochastic Processes and Control for Jump-Diffusions: Modeling, Analysis and Computation. 1st Edn., SIAM Books, ISBN: 0898716330, 978-0898716337, pp: 443.

Heston, S.L., 1993. A closed-form solution for options with stochastic volatility with applications to bond and currency options. Rev. Financial Stud., 6: 327343. DOI: $10.1093 / \mathrm{rfs} / 6.2 .327$

Intarasit, A. and P. Sattayatham, 2010. A geometric Brownian motion model with compound Poisson process and fractional stochastic volatility. Adv. Appli. $\quad$ Stat., $16:$ 25-47. http://risklabbkk.com/images/PDF_files/Intarasit01 .pdf

Khaled, K. and M. Samia, 2010. Estimation of the parameters of the stochastic differential equations black-scholes model share price of gold. J. Math. Stat., 6 : $\quad 421-424 . \quad$ DOI: 10.3844/jmssp.2010.421.424

Magnus, F.J. and O.E. Fosu, 2006. Modeling and forecasting volatility of returns on the ghana stock exchange using garch models. Am. J. Applied Sci., 3: 2042-2048. DOI: 10.3844/ajassp.2006.2042.2048

Shamiri, A. and Z. Isa, 2009. Modeling and forecasting volatility of the malaysian stock markets. J. Math. Stat., 5 : 234-240. 10.3844/jmssp.2009.234.240

Thao, T.H., 2006. An approximate approach to fractional analysis for finance. Nonlinear Anal.: Real World Appli., 7: 124-132. DOI: 10.1016/J.NONRWA.2004.08.012 